\title{
Maximal Voluntary Fingertip Force Production Is Not Limited by Movement Speed in Combined Motion and Force Tasks
}

\author{
Kevin G. Keenan, ${ }^{2,3,6}$ Veronica J. Santos, ${ }^{4,6}$ Madhusudhan Venkadesan, ${ }^{5,6}$ and Francisco J. Valero-Cuevas ${ }^{1,2,6}$ \\ ${ }^{1}$ Department of Biomedical Engineering and 2Division of Biokinesiology and Physical Therapy, University of Southern California, Los Angeles, California \\ 90089, ${ }^{3}$ Department of Human Movement Science, University of Wisconsin-Milwaukee, Milwaukee, Wisconsin 53211, ${ }^{4}$ Department of Mechanical and \\ Aerospace Engineering, Arizona State University, Tempe, Arizona 85287, ${ }^{5}$ School of Engineering and Applied Sciences, Harvard University, Cambridge, \\ Massachusetts 02138, and ${ }^{6}$ Sibley School of Mechanical and Aerospace Engineering, Cornell University, Ithaca, New York 14853
}

Numerous studies of limbs and fingers propose that force-velocity properties of muscle limit maximal voluntary force production during anisometric tasks, i.e., when muscles are shortening or lengthening. Although this proposition appears logical, our study on the simultaneous production of fingertip motion and force disagrees with this commonly held notion. We asked eight consenting adults to use their dominant index fingertip to maximize voluntary downward force against a horizontal surface at specific postures (static trials), and also during an anisometric "scratching" task of rhythmically moving the fingertip along a $5.8 \pm 0.5 \mathrm{~cm}$ target line. The metronome-timed flexion- extension movement speed varied 36-fold from "slow" ( $1.0 \pm 0.5 \mathrm{~cm} / \mathrm{s})$ to "fast" $(35.9 \pm 7.8 \mathrm{~cm} / \mathrm{s})$. As expected, maximal downward voluntary force diminished $(44.8 \pm 15.6 \% ; p=0.001)$ when any motion (slow or fast) was added to the task. Surprisingly, however, a 36-fold increase in speed did not affect this reduction in force magnitude. These remarkable results for such an ordinary task challenge the dominant role often attributed to force-velocity properties of muscle and provide insight into neuromechanical interactions. We propose an explanation that the simultaneous enforcement of mechanical constraints for motion and force reduces the set of feasible motor commands sufficiently so that force-velocity properties cease to be the force-limiting factor. While additional work is necessary to reveal the governing mechanisms, the dramatic influence that the simultaneous enforcement of motion and force constraints has on force output begins to explain the vulnerability of dexterous function to development, aging, and even mild neuromuscular pathology.

\section{Introduction}

Force-velocity properties of muscle are generally considered a critical limiting factor for maximal voluntary force production during anisometric tasks, i.e., when muscles are shortening or lengthening. Given that skeletal muscle is the primary actuator for generating force and movement, its force-velocity properties (Hill, 1938; Katz, 1939), as well as its force-length properties (Ralston et al., 1947; Gordon et al., 1966), undoubtedly affect voluntary force production, as repeatedly shown by biomechanical models (Hoy et al., 1990; Cheng et al., 2000) and experimental studies (Westling et al., 1990; Lieber et al., 1994; Valero-Cuevas et al., 1998). In the specific context of finger function, most exper-

\footnotetext{
Received Feb. 19, 2009; revised May 18, 2009; accepted May 18, 2009.

This work was supported in part by the Whitaker Foundation, National Science Foundation (NSF) Grants 0312271 and 0237258, and National Institutes of Health (NIH) Grants HD048566, AR050520, and AR052345 (to F.J.V.-C.). V.J.S. was partially supported by an NSF Graduate Research Fellowship. M.V. was supported by the NSF Frontiers in Integrative Biological Research Grant 0425878 during manuscript preparation. The contents of this manuscript are solely the responsibility of the authors and do not necessarily represent the official views of the National Institute of Arthritis and Musculoskeletal and Skin Diseases, the National Institute of Childhood and Human Development, the $\mathrm{NIH}$, the Whitaker Foundation, or the NSF.

Correspondence should be addressed to Francisco J. Valero-Cuevas, University of Southern California, $3710 \mathrm{MC}$ Clintock Avenue, RTH 404, Los Angeles, CA 90089-2905. E-mail: valero@usc.edu. D0I:10.1523/JNEUROSCI.0853-09.2009

Copyright $\odot 2009$ Society for Neuroscience $\quad$ 0270-6474/09/298784-06\$15.00/0
}

iments examine either force or motion tasks separately [e.g., Valero-Cuevas et al. (1998), Carson and Riek (2001), Shinohara et al. (2005), and Cole (2006)]. In this study, we test the hypothesis that force-velocity properties of muscle are a limiting factor for voluntary force production during more "ecological" and "complex" manipulation tasks requiring simultaneous production of both fingertip motion and force. We tested this by instructing subjects to maximize voluntary downward force into a low-friction Teflon surface during static contractions at different positions along the surface and while flexing and extending the index fingertip along the surface at slow to fast speeds. We expected to find that maximal voluntary downward force would scale with movement speed, consistent with force-velocity properties of muscle. Surprisingly, voluntary force production was independent of movement speed. A preliminary account of our findings was published in abstract form (Keenan et al., 2007).

\section{Materials and Methods}

Eight subjects (mean age: $22.8 \pm 2.8$ years, range: $19-27$ years, five men) provided written informed consent before participating in the study and the Committee on Human Subjects Research at Cornell University approved the procedures of the study. All subjects reported being healthy without any known neurological problems or hand pathologies, and 


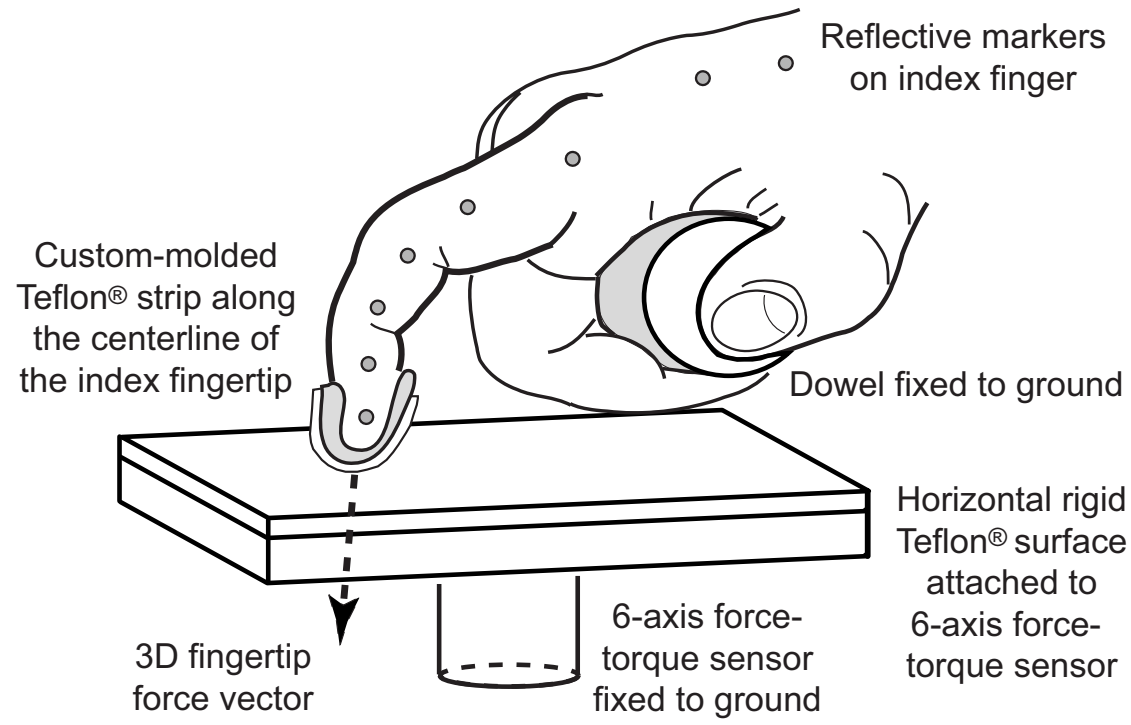

Figure 1. Experimental setup. Subjects were instructed to maximize downward force against a horizontal low-friction Teflon surface, first during a brief maximal voluntary static contraction at one of three starting positions, and then while flexing and extending their right index fingertip along the surface at one of three movement speeds.

were right-hand dominant [Edinburgh Handedness Inventory (Oldfield, 1971)].

Experimental arrangement. Subjects had their right forearm strapped to a horizontal platform with a vacuum foam pad (Versaform pillow, Tumble Forms) to immobilize the elbow and forearm. Subjects grasped a horizontal dowel with all fingers and thumb, except for the index finger, which was free to move so as to slide on a low-friction Teflon surface (Fig. 1). The Teflon surface was attached to a rectangular pedestal mounted on a six-axis force-torque sensor (model 20E12A-I25, JR3) that measured forces at $1000 \mathrm{~Hz}$. Surface position and height were adjusted such that the index finger was in a neutral ad-abduction posture and could flex and extend completely across the pedestal. Subjects wore a custom-molded cover (i.e., thermoplastic material with rubber mesh insert for comfort) on the fingertip, with a thin Teflon strip secured along the centerline of the index fingertip (Fig. 1). This custom-molded cover with the attached Teflon strip (1) helped remove the discontinuity at the fingernail, (2) enforced the fingertip force vector to remain oriented close to the surface normal, and (3) provided minimal resistance to sliding at any speed (Teflon-Teflon friction coefficient $\approx 0.04$ ). To accommodate simultaneous motion and force production, the fingertip cover in this study is a modified version of those used in previous studies of static force production (Valero-Cuevas et al., 1998; Valero-Cuevas, 2000; Venkadesan and Valero-Cuevas, 2008). We also recorded the 3D location of the index finger at $200 \mathrm{~Hz}$ using a five-camera motion-capture system (Vicon Peak) that tracked eight reflective markers along the radial aspect of the finger (Fig. 1). The contact point of the index finger along the Teflon surface was identified from the most distal reflective marker placed on the index finger, which moved in a plane nearly parallel with the horizontal Teflon surface.

Experimental procedure. Each experiment consisted of 18 trials ( 3 starting positions $\times 3$ movement speeds $\times 2$ trials for each condition). For each trial subjects were instructed to maximize voluntary downward force, first during a brief maximal voluntary static contraction at one of three starting positions, and then while flexing and extending their right index fingertip along the Teflon surface at one of three movement speeds (Fig. 2). Thus, each trial consisted of a static and dynamic component. We block randomized the experimental trials by movement speed (i.e., slow, moderate, and fast) to improve motion repeatability. For each movement speed, subjects were given at least two trials to practice the task. Subjects were instructed to, as a first priority, produce a maximal voluntary downward force. Additionally, during the dynamic component of each trial, subjects were instructed to continue producing a maximal downward force along with an accurate and smooth finger move- ment along the target line. This ensured that the explicit motor control goal of producing maximal voluntary downward force simultaneously with smooth motion remained consistent across all trials. Ensuring that the motion was performed smoothly was necessary to prevent slipping across the low-friction surface. The criteria for selecting the two experimental trials for analysis were as follows: (1) the absence of slipping (identified by one investigator visually and verified by motion capture and force data), and (2) confirmation by the subject verbally that voluntary force production was indeed maximal for both static and dynamic components of each trial.

The three starting positions were customized for differences in hand size by having subjects fully flex and extend their right index fingertip along a target line on the horizontal Teflon surface (Fig. 2A,B, dashed purple line). The bounds of this target line were determined from the fully extended and flexed finger postures and the three positions were marked at equal intervals along the target line (Fig. $2 \mathrm{~A}$, positions 1,2 , and 3 at 25,50 , and $75 \%$ along the target line, respectively). Finger movement lengths were $\sim 5.8 \pm 0.5 \mathrm{~cm}$ (mean $\pm \mathrm{SD})$. Subjects performed six trials starting at each of the three positions. Thus, there were six maximal voluntary static contractions performed at each starting position (Fig. $2 B$, red $\times$ ). Because there was slight variability in the exact location along the target line where maximal voluntary static force occurred (Fig. 2 B; red $\times$ ), bounds were set for each of the three positions based on the position along the target line where maximal voluntary static forces occurred (Fig. $2 \mathrm{~B}$, blue regions).

The three movement speeds (Fig. 2C) were as follows: (1) slow: moving as slowly as possible in the direction of flexion along the target line; (2) moderate: cadence period $=2.2 \mathrm{~s}$; and (3) fast: cadence period $=$ $0.6 \mathrm{~s}$. For the slow movement speed, only flexion was performed after the maximal static contraction to prevent fatigue. Also, the slow movement speed $(\sim 1 \mathrm{~cm} / \mathrm{s})$ was chosen to ensure that muscle shortening velocities were performed at velocities close to isometric on the force-velocity relationship (Lieber and Bodine-Fowler, 1993). For the moderate and fast movement speeds, a metronome train of 12 tones indicated the time when the finger should reverse direction to accomplish six equalduration cycles of flexion-extension movement. Rest periods of at least $60 \mathrm{~s}$ between trials were used to prevent fatigue.

Data analysis. Data were acquired with Vicon Workstation software (version 4.6; Vicon Peak) and analyzed off-line using custom-written programs in Matlab (version 7.1, The MathWorks). We low-pass filtered ( $40 \mathrm{~Hz}$ cutoff) the force data from all three force axes before calculating the Euclidean magnitude of the force vector to quantify force magnitude. Maximal voluntary downward forces were calculated for each subject at positions 1, 2, and 3 for each of the four movement conditions (static, slow, moderate, and fast). To compare forces across subjects and account for differences in scale, forces were also normalized to the largest maximal voluntary force value measured across all conditions for each subject.

We used repeated-measures ANOVA (SAS version 9.1.3) to test for differences in maximal force across movement condition (four levels of velocity) and position (three levels of posture) along the target line. We verified that the residuals were normally and identically distributed, applied a Tukey-Kramer adjustment for multiple comparisons when reporting $p$-values, and set the significance level for all statistical tests at 0.05 . Results are reported as means \pm SDs in the text and means \pm SEs in Figure 3.

\section{Results}

Figure 3 shows the absolute and normalized maximal voluntary downward forces (Fig. 3; mean \pm SE, $n=8$ ) for three positions 
along the target line and four movement conditions. The largest maximal force measured across all conditions occurred during static contractions performed at either position 1 or position 2 (Fig. $3 A$ ). Forces across all conditions were normalized to the maximal force that was measured during the static contractions. As the results of statistical comparisons were qualitatively identical for absolute and normalized forces, we report the findings for normalized forces only (Fig. 3B).

As expected, maximal voluntary downward force diminished (44.8 $\pm 15.6 \%$ maximal static force; $p<0.001$ ) when motion was added to the task (Fig. $3 B$ ). Remarkably, there were no significant differences across normalized force magnitudes $(p<0.001)$ between slow $(55.7 \pm 16.6 \%)$, moderate $(56.1 \pm 13.5 \%)$, and fast $(53.9 \pm$ $17.5 \%$ ) movement speeds (Fig. $3 B$ ). Surprisingly, there were no significant differences in maximal force even though movement speeds varied 36-fold, from slow $(1.0 \pm 0.5 \mathrm{~cm} / \mathrm{s})$ to fast $(35.9 \pm 7.8 \mathrm{~cm} / \mathrm{s})$.

The position of the fingertip influenced maximal voluntary force, as maximal normalized forces at positions 1 (70.5 $21.4 \%)$ and $2(64.3 \pm 19.8 \%)$ were significantly greater than normalized force at position $3(53.8 \pm 18.2 \% ; p=0.0011$ and $p=0.0125$, respectively) (Fig. $3 B$ ). The normalized forces above are collapsed across all four movement speeds (i.e., one static contraction and the three movement speeds). There was a significant interaction between movement condition and position $(p=0.0409)$, with static contractions at positions 1 and 2 greater $(p<$ $0.001)$ than all other conditions, though no significant interactions existed for all other comparisons $(p>0.81)$. Thus, there were no significant differences in maximal force across movement speeds for the three different positions. We also tested whether direction of movement influenced maximal voluntary anisometric force (Fig. 3B). Maximal force during the flexion phase of the movement (55.0 \pm $4.9 \%)$ was greater $(p=0.0001)$ than during the extension phase $(37.1 \pm 1.3 \%)$.

Note that because the slow movement condition involved only flexion (no extension to prevent fatigue), no data were obtained from position 1 at the slow speed (Fig. $3 A, B$ ) or during the extension phase of movement (Fig. 3B).

\section{Discussion}

We find that maximal voluntary fingertip force is insensitive to finger movement velocity. This challenges the common hypothesis that the force-velocity properties of muscle are a primary limiting factor of force output during anisometric tasks. Therefore, it is necessary to consider other limiting factors, such as how the musculoskeletal structure of the fingers, the constraints of the

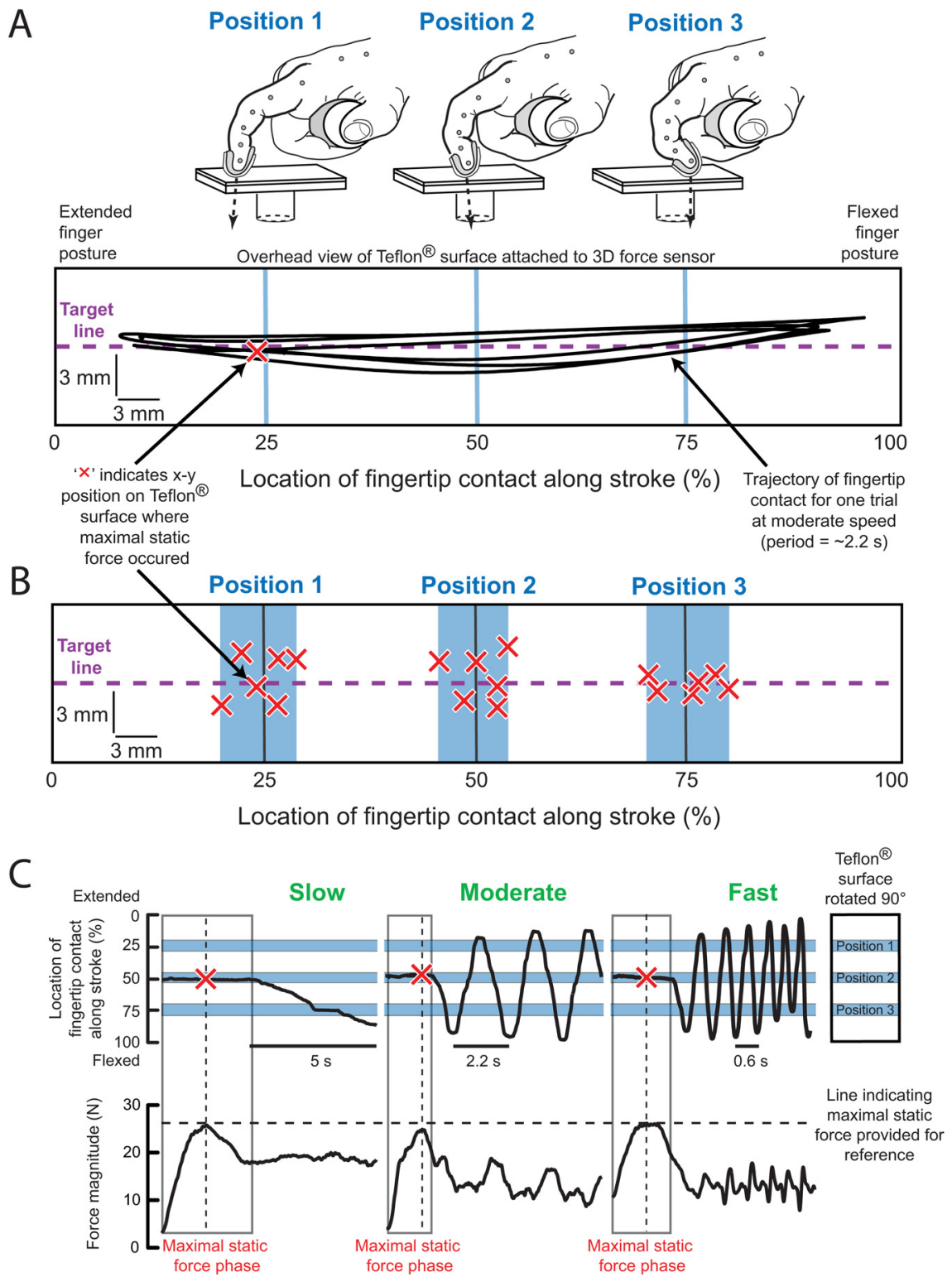

Figure 2. Representative data from one subject. $\boldsymbol{A}$, The three starting positions (blue) were at 25,50 , and $75 \%$ along a target line (purple) on the horizontal Teflon surface. The black trace shows a trajectory along the Teflon surface during one trial at moderate speed. The red $\times$ marks the location where maximal static force occurred for a trial starting at position 1 . $\boldsymbol{B}$, Subjects performed a total of 18 trials, with six trials starting at each of the three positions. Thus, six maximal voluntary static contractions were performed at each of the three starting positions (red $\times$ ). Bounds were set for each position based on the location along the target line where maximal static forces occurred (blue regions). $C$, Representative position (top trace) and force (bottom trace) data are shown for three trials starting at position 2. The three movement speeds following the maximal static force phase (red) were as follows: (1) as slow as possible; (2) moderate: cadence period $=2.2 \mathrm{~s}$; and ( 3 ) fast: cadence period $=0.6$ s. Note that for the slow condition, only flexion was performed after the maximal static contraction to prevent fatigue.

task, and the nature of the neural controller conspire to reduce motor output even for ordinary manipulation tasks that combine motion and force production. As we argue below, these results suggest that our musculature is not redundant in the context of combined motion and force tasks, and may begin to explain the vulnerability of dexterous function to development, aging, and even mild neuromuscular pathology.

Changes in maximal voluntary downward force in the static case are consistent with finger mechanics and force-length properties of muscle (Fig. 3). Changes in finger posture alters muscle fiber length and, in turn, the transformation of muscle forces into finger forces (Zajac, 1992; Valero-Cuevas et al., 1998; Valero- 


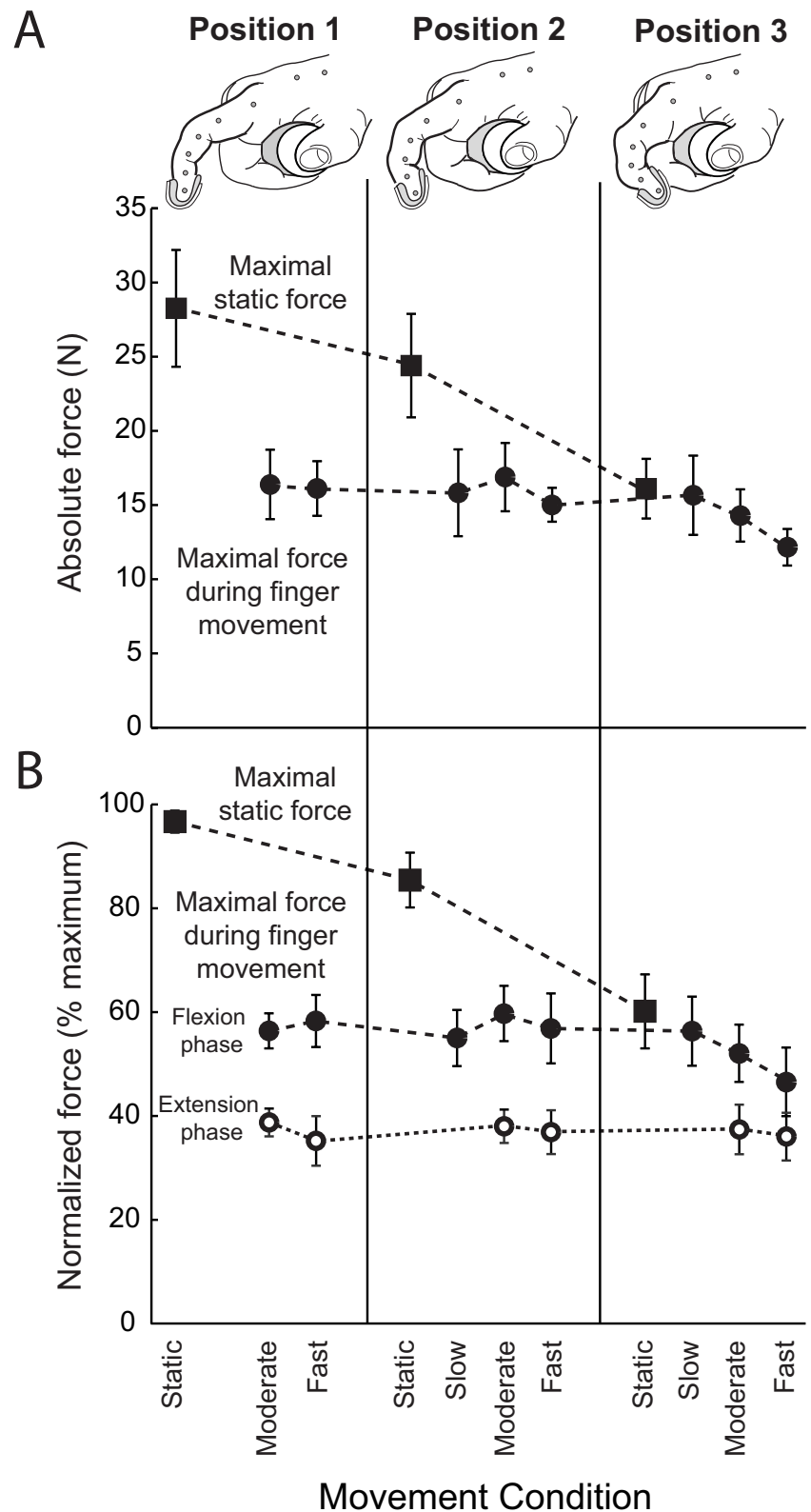

Figure 3. Summary data from all subjects $(n=8)$. Maximal downward force diminished when motion was added to the task; however, the maximal force with motion was not affected by movement speed or position along the target line. $\boldsymbol{A}, \boldsymbol{B}$, Absolute $(\boldsymbol{A})$ and normalized $(\boldsymbol{B})$ maximal downward forces (mean $\pm \mathrm{SE}$ ) are shown for static (circles) and dynamic anisometric (squares) trials. Forces were normalized to the largest maximal force value measured across all conditions for each subject (i.e., static forces at position 1 or 2). Maximal forces across all movement speeds and static forces at position 3 were reduced with respect to static forces at position 1 or 2 . In addition, we examined whether the direction of movement influenced force production. Force during the flexion phase was greater than during the extension phase $(\boldsymbol{B})$. All differences were statistically significant ( $p=0.0001$ ). Note that because the slow movement condition involved only flexion (no extension), no data were obtained from position 1 at the slow speed $(\boldsymbol{A}$ or $\boldsymbol{B})$ or during the extension phase of movement $(\boldsymbol{B})$.

Cuevas, 2000, 2009). In addition, the reduction in maximal voluntary downward force in the anisometric case, relative to static isometric contractions, could arise from the force-velocity properties of muscle. However, force-velocity properties alone cannot explain our observations in the anisometric case, because maximal downward force was insensitive to movement speed. If force-velocity properties limited the maximal voluntary force in this task, voluntary force magnitude would have decreased with increasing fingertip velocities due to the large changes in sarcomere velocities that accompany finger flexion-extension (An et al., 1983; Lieber et al., 1992; Zajac, 1992). Remarkably, maximal force magnitude remained relatively unchanged when fingertip velocities varied over a 36-fold range (Fig. 3). The differences in voluntary force magnitude between the flexion and extension phases are of interest, and may have resulted from differences in the muscle coordination patterns needed to move the finger in opposite directions. Nevertheless, the force magnitude remained insensitive to change in movement speed for each movement direction. Also, if force-length properties were a limiting factor, as in the static condition and earlier work (Valero-Cuevas et al., 1998; Valero-Cuevas, 2000), then postural changes could have affected the maximal fingertip force. Note that because posture affects the mechanical transmission of muscle forces independently of - and in addition to-muscle force-length effects (Valero-Cuevas et al., 1998; Valero-Cuevas, 2009), we cannot disambiguate their compounded effects during the isometric trials.

\section{Idiosyncratic performance across subjects}

Subjects may have lacked the motivation or skill to perform the task to their biomechanical limits. As with all studies involving voluntary contractions, the potential confounds of skill, practice, and learning cannot be completely eliminated. Nonetheless, reasons for disregarding idiosyncratic behavioral limitations as the explanation include the following: (1) our task resembles many everyday tasks combining motion and large fingertip forces: scratching, peeling fruit, etc.; (2) subjects were extremely motivated and quickly learned to perform well; (3) practice trials ensured at least two successful trials for each combination of movement condition and position; (4) subjects reported that they could not produce any higher force; and (5) the results were consistent across all subjects and for two repeated trials for each task condition.

\section{Muscle properties}

Nonlinearities of the force-velocity curve at the isometric point and history-dependent effects such as force enhancement (Herzog et al., 2006) could potentially be exploited to enhance force production during the static case but not during motion, thus explaining the dramatic drop in force production even between the static and slow-motion cases. For example, small oscillations around the nominal finger posture in the static case could result in muscles oscillating quickly between producing force eccentrically and concentrically. Small oscillations and reversal of movement direction together with force enhancement (a historydependent muscle property) may increase maximal static force. However, these effects at best explain the drop in maximal force between static and slow-motion conditions, or between flexion and extension phases, but not the insensitivity to an $\sim 36$-fold range in fingertip movement speed.

Having excluded behavioral or muscle properties as the predominant mechanism underlying our results, we propose two alternate explanations to be explored in future work.

\section{Musculoskeletal biomechanics}

Limbs and fingers are naturally stronger in some directions than others, as described by their feasible force set (Milner and Franklin, 1998; Valero-Cuevas et al., 1998; Yokogawa and Hara, 2002; Valero-Cuevas, 2009), a consequence of musculoskeletal geometry and muscle parameters. The static and anisometric conditions require the fingertip force vector to be directed slightly 
differently: inside and outside the small friction cone, respectively. It is possible that the anisometric task induces mechanical changes in the feasible force set such that maximal forces appear insensitive to the changes in muscle fiber lengths and velocities. We regard this explanation as unlikely because of the small friction cone $\left(\sim 2^{\circ}\right)$, but future musculoskeletal modeling work is necessary to test it. In addition, it is interesting that for one of the three finger postures (position 3), maximal voluntary force attained during the maximal static force production was similar to that exerted during finger movement (Fig. 3). It is conceivable that subjects voluntarily adopted a coordination pattern to generate a force that could be maintained across all postures, regardless of movement condition. However, for the slow movement speed, it is unclear why they would adopt such a strategy when moving from position 1 to 2 , although future work is necessary to test it.

\section{Superimposition of task constraints}

In our opinion, the more likely explanation is that the simultaneous enforcement of motion and force task constraints by the neural controller reduces the set of feasible motor commands to the point that it overrides force-velocity properties of muscle as a limiting factor. The initial change in force before the onset of motion (Fig. 2) is explained simply by the need to change muscle coordination patterns from that for maximal voluntary isometric force to that which can produce both motion and force (Venkadesan and Valero-Cuevas, 2008). Call the set of all possible combinations of muscle activations that the CNS can command the "motor command set" (Valero-Cuevas et al., 1998; ValeroCuevas, 2009). There is some subset of this motor command set that can successfully achieve a given task; call it the feasible command subset. Having more muscles than joint degrees of freedom (i.e., muscle redundancy) simply means that this subset is generally not a single point, but a continuum. For the static case, there is a feasible command subset of all combinations of muscle forces that produce well directed downward fingertip force vectors (Valero-Cuevas et al., 1998; Valero-Cuevas, 2000). In general, there exists a unique combination of muscle forces (a point within the feasible command subset) that maximizes fingertip force within the friction cone (Valero-Cuevas et al., 1998). However, moving the finger parallel to the surface has its own feasible command subset different from that for isometric force (Yoshikawa et al., 1990; Venkadesan and Valero-Cuevas, 2008, 2009). Simultaneously producing both vertical fingertip force and horizontal fingertip motion can only be achieved by points in the intersection of their corresponding feasible command subsets. While such a task decomposes force and motion along Cartesian orthogonal axes, the corresponding representations are not necessarily orthogonal in joint, muscle, and activation coordinate systems given the nonlinear transformations due to postural changes (Valero-Cuevas, 2009). The feasible command subsets emerge from, and are defined by, the interactions among the biomechanical and musculoskeletal structure of the system, the mechanical constraints of the task, muscle properties, series elastic elements, sensorimotor pathways, and the neural control strategy implemented by the nervous system. This subset of feasible motor commands is referred to by various names including task-specific muscle synergy (Scholz and Schöner, 1999), the feasible activation set or manifold of feasible activations (ValeroCuevas et al., 1998; Venkadesan and Valero-Cuevas, 2008; Valero-Cuevas, 2009), the set over which to optimize motor commands (Loeb, 2000; Todorov, 2004), a basis for muscle synergies (Tresch et al., 1999; d'Avella et al., 2006), and so on. Re- gardless of the different nomenclature, these descriptors all share a fundamental tenet. Namely, not all motor commands are able to perform a task, but rather there exists a well defined subset of feasible motor commands from which the nervous system must choose a specific command.

In this context, our proposed explanation of the results is that the intersection between the respective subsets for the production of motion and force does not contain points where the muscles can be maximally activated, and thus force-velocity properties do not limit maximal fingertip force. Said differently, the features of the task and neural controller-and not the force-velocity properties of the muscles - are the "active constraints" defining the boundary of the feasible output of the system (Gill and Murray, 1981; Chvátal, 1983). This interpretation supports the notion that the superimposition of task constraints can severely reduce the set of feasible motor commands as proposed by Loeb (2000), and is complementary to recent demonstration of important differences and nuances in the control of fingertip motion and force (Mah and Mussa-Ivaldi, 2003; Venkadesan and Valero-Cuevas, 2008). Note that in the absence of a mathematical model and further experimental work, it is not possible to unambiguously identify the governing mechanism(s) underlying our results, and we only conclude that the force-velocity characteristic of muscle is not the primary limiting factor in this task.

Finally, our results and proposed explanation may clarify the apparent and long-standing paradox between the concept of muscle redundancy and the clinical reality of motor development and dysfunction. If, for example, hand musculature is so redundant, why then is manipulation so vulnerable to developmental problems (Forssberg et al., 1991), mild neurological pathologies, and aging (Schreuders et al., 2006)? This apparent inconsistency may arise simply because experiments and models often use simplified tasks for which the musculature is indeed redundant. In contrast, natural behavior often involves tasks that combine multiple goals or transitions between goals (Loeb, 2000). This work and recent studies (Venkadesan and Valero-Cuevas, 2008, 2009) indicate that even ordinary manipulation tasks can push the neuromuscular system to its limit of performance when requiring combinations of, or transitions between, motion and force production. Further investigations are necessary to better understand how the neuromuscular system robustly meets the requirements for natural behavior, and how development, aging, and mild neurological pathology affect dexterous manipulation.

\section{References}

An KN, Ueba Y, Chao EY, Cooney WP, Linscheid RL (1983) Tendon excursion and moment arm of index finger muscles. J Biomech 16:419-425.

Carson RG, Riek S (2001) Changes in muscle recruitment patterns during skill acquisition. Exp Brain Res 138:71-87.

Cheng EJ, Brown IE, Loeb GE (2000) Virtual muscle: a computational approach to understanding the effects of muscle properties on motor control. J Neurosci Methods 101:117-130.

Chvátal V (1983) Linear programming. San Francisco: Freeman.

Cole KJ (2006) Age-related directional bias of fingertip force. Exp Brain Res 175:285-291.

d'Avella A, Portone A, Fernandez L, Lacquaniti F (2006) Control of fastreaching movements by muscle synergy combinations. J Neurosci 26:7791-7810.

Forssberg H, Eliasson AC, Kinoshita H, Johansson RS, Westling G (1991) Development of human precision grip I: basic coordination of force. Exp Brain Res 85:451-457.

Gill P, Murray W (1981) Practical optimization. London: Academic.

Gordon AM, Huxley AF, Julian FJ (1966) The variation in isometric tension with sarcomere length in vertebrate muscle fibres. J Physiol 184:170-192.

Herzog W, Lee EJ, Rassier DE (2006) Residual force enhancement in skeletal muscle. J Physiol 574:635-642. 
Hill AV (1938) The heat of shortening and the dynamic constants of muscle. Proc R Soc Lond B Biol Sci 126:136-195.

Hoy MG, Zajac FE, Gordon ME (1990) A musculoskeletal model of the human lower extremity: the effect of muscle, tendon, and moment arm on the moment-angle relationship of musculotendon actuators at the hip, knee, and ankle. J Biomech 23:157-169.

Katz B (1939) The relation between force and speed during muscular contraction. J Physiol 96:45-64.

Keenan KG, Ashley D, McNamara III RV, Song S, Kanar T, Valero-Cuevas FJ (2007) Muscle mechanics do not suffice to explain the reduction in maximal voluntary fingertip force in combined motion and force tasks. Soc Neurosci Abstr 33:81.13/KK86.

Lieber RL, Bodine-Fowler SC (1993) Skeletal muscle mechanics: implications for rehabilitation. Phys Ther 73:844-856.

Lieber RL, Jacobson MD, Fazeli BM, Abrams RA, Botte MJ (1992) Architecture of selected muscles of the arm and forearm: anatomy and implications for tendon transfer. J Hand Surg [Am] 17:787-798.

Lieber RL, Loren GJ, Fridén J (1994) In vivo measurement of human wrist extensor muscle sarcomere length changes. J Neurophysiol 71:874-881.

Loeb GE (2000) Overcomplete musculature or underspecified tasks? Motor Control 4:81-83; discussion 97-116.

Mah CD, Mussa-Ivaldi FA (2003) Evidence for a specific internal representation of motion-force relationships during object manipulation. Biol Cybern 88:60-72.

Milner TE, Franklin DW (1998) Characterization of multijoint finger stiffness: dependence on finger posture and force direction. IEEE Trans Biomed Eng 45:1363-1375.

Oldfield RC (1971) The assessment and analysis of handedness: the Edinburgh inventory. Neuropsychologia 9:97-113.

Ralston HJ, Inman VT, Strait LA, Shaffrath MD (1947) Mechanics of human isolated voluntary muscle. J Physiol 65:2687-2695.

Scholz JP, Schöner G (1999) The uncontrolled manifold concept: identifying control variables for a functional task. Exp Brain Res 126:289-306.

Schreuders TA, Selles RW, Roebroeck ME, Stam HJ (2006) Strength measurements of the intrinsic hand muscles: a review of the development and evaluation of the Rotterdam intrinsic hand myometer. J Hand Ther 19: 393-401; quiz 402.

Shinohara M, Keenan KG, Enoka RM (2005) Fluctuations in motor output during steady contractions are weakly related across contraction types and between hands. Muscle Nerve 31:741-750.

Todorov E (2004) Optimality principles in sensorimotor control. Nat Neurosci 7:907-915.

Tresch MC, Saltiel P, Bizzi E (1999) The construction of movement by the spinal cord. Nat Neurosci 2:162-167.

Valero-Cuevas FJ (2000) Predictive modulation of muscle coordination pattern magnitude scales fingertip force magnitude over the voluntary range. J Neurophysiol 83:1469-1479.

Valero-Cuevas FJ (2009) A mathematical approach to the mechanical capabilities of limbs and fingers. Adv Exp Med Biol 629:619-633.

Valero-Cuevas FJ, Zajac FE, Burgar CG (1998) Large index-fingertip forces are produced by subject-independent patterns of muscle excitation. J Biomech 31:693-703.

Venkadesan M, Valero-Cuevas FJ (2008) Neural control of motion-to-force transitions with the fingertip. J Neurosci 28:1366-1373.

Venkadesan M, Valero-Cuevas FJ (2009) Effects of neuromuscular lags on controlling contact transitions. Philos Transact A Math Phys Eng Sci 367:1163-1179.

Westling G, Johansson RS, Thomas CK, Bigland-Ritchie B (1990) Measurement of contractile and electrical properties of single human thenar motor units in response to intraneural motor-axon stimulation. J Neurophysiol 64:1331-1338.

Yokogawa R, Hara K (2002) Measurement of distribution of maximum index-fingertip force in all directions at fingertip in flexion/extension plane. J Biomech Eng 124:302-307.

Yoshikawa T, Harada K, Matsumoto A (1990) Hybrid position/force control of flexible-macro/rigid-micro manipulator systems. IEEE Trans Rob Autom 12:633-640.

Zajac FE (1992) How musculotendon architecture and joint geometry affect the capacity of muscles to move and exert force on objects: a review with application to arm and forearm tendon transfer design. J Hand Surg [Am] 17:799-804. 